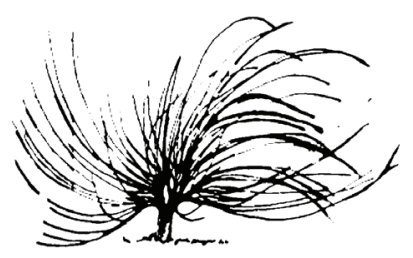

\title{
EI aprendizaje basado en la investigación (ABI) como un factor para el fortalecimiento de los programas educativos de la Universidad Quintana Roo en Playa del Carmen, México
}

\author{
Mariana Figueroa de la Fuente ${ }^{1}$ \\ Universidad de Quintana Roo \\ Playa del Carmen, México \\ mfigueroa@uqroo.edu.mx \\ David Reyes Coronado 2 \\ Universidad de Quintana Roo \\ Playa del Carmen, México \\ davrey@uqroo.edu.mx \\ Natalia Fiorentini Cañedo ${ }^{3}$ \\ Universidad de Quintana Roo \\ Playa del Carmen, México \\ fiorentini@uqroo.edu.mx
}

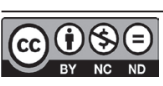

Recibido: 28 de febrero de 2017-Aprobado: 21 de mayo de 2018

http://dx.doi.org/10.15359/rep.13-1.6

1 Maestra en Tecnología Educativa del Tecnológico de Monterrey. Doctoranda en Liderazgo y dirección de Instituciones de educación Superior por la Universidad Anáhuac del Norte. Y profesora investigadora de la Universidad Quintana Roo en Playa del Carmen, México.

2 David Reyes Coronado es Doctor en Ciencias por el Centro de Investigación y Estudios Avanzados (CINVESTAV) del Instituto Politécnico Nacional de México. Actualmente se desempeña como profesor-investigador en la Universidad de Quintana Roo, imparte asignaturas de: matemáticas, física, métodos y técnicas de investigación, seminario de titulación.

3 Historiadora. Doctora en Historia por la Universidad Nacional Autónoma de México. Profesora-investigadora de la Unidad Académica Playa del Carmen de la Universidad de Quintana Roo, México. 


\title{
Resumen
}

El presente trabajo analiza las características del aprendizaje basado en investigación (ABI), con el objetivo de abordar cómo la articulación de la docencia con la investigación puede ser un factor para el fortalecimiento de los programas educativos en educación superior, concretamente en la Universidad de Quintana Roo, Unidad Académica Playa del Carmen (UAPC). Para ello, con un enfoque mixto de investigación se efectuó la revisión de documentos significativos y bibliografía especializada como técnicas cualitativas iniciales. El análisis de esta fase contribuyó al diseño de un cuestionario estructurado para llevar a cabo una encuesta en la parte cuantitativa, que permitió conocer la disposición y los obstáculos para implementar el ABI. Entre los principales hallazgos destacan el interés del profesorado por fortalecer sus capacidades investigativas, capacitarse en conocimientos teóricos sobre el ABI e incluir al alumnado en las investigaciones, para poder con todo ello hacer frente a la necesidad de formar profesionistas con creatividad; con capacidad de analizar, pensar críticamente, escribir y expresarse con eficacia; resolver problemas complejos, y sintetizar, entre otros retos de la realidad educativa del siglo XXI.

Palabras clave: Aprendizaje basado en la investigación, calidad educativa, articulación docencia-investigación, educación superior.

\begin{abstract}
This paper analyzes the characteristics of ResearchBased Learning (RBL) with the aim of understanding how the articulation of teaching and the research process can be a factor for strengthening higher education, specifically at the University of Quintana Roo, Playa del Carmen Academic Unit (UAPC). To do this, significant documents and literature were revised using qualitative techniques. This phase contributed to the design of a structured questionnaire to carry out a survey which allowed knowing the disposition and the obstacles to implement the RBL at UAPC. Among the findings, the following stand out: the interest of the faculty members to strengthen its investigative capabilities,
\end{abstract}


training in theoretical knowledge about the RBL, and the involvement of students in research in order to be able to cope with the need for creative professionals with a capacity to analyze, to think critically, write and express themselves effectively, solve complex problems, synthesize, among other challenges of the educational reality of the 21 st Century.

Keywords: Research-Based Learning, educational quality, teaching-research, higher education

\section{Introducción}

La Unidad Académica Playa del Carmen (UAPC), ubicada en la cabecera del municipio Solidaridad, se creó en el año 2009 como una respuesta de la propia Universidad de Quintana Roo (UQROO) y de las autoridades federales y estatales a la baja cobertura educativa en el nivel superior en la entidad. De hecho, en 2012, Quintana Roo, Chiapas, Oaxaca y Guerrero continuaban siendo los Estados con los niveles más bajos de cobertura con cifras menores al 20\% (UNAM, 2012). ${ }^{4}$ Concretamente en el Municipio de Solidaridad, las últimas cifras oficiales refieren la existencia de 2,158 estudiantes de universidad en modalidad escolarizada (SEP, 2015). De este grupo, 639 (30\%) estudian en la UAPC (UQROO-DGP, 2016), lo que habla del importante papel que tiene la UQROO en el municipio, no solo en materia de cobertura educativa, sino en la generación de profesionistas que respondan a las demandas sociales, culturales y laborales de la región. Cabe recordar que la UAPC tiene como misión:

Formar excelentes personas comprometidas con el progreso del Estado de Quintana Roo y México, a través de la generación de conocimiento y del fomento de valores, actitudes y habilidades relevantes y emprendedoras, que les permita insertarse a un entorno de mayor competencia, así como coadyuvar a alcanzar a un desarrollo sustentable. (UQROO-SIGC, s. f., p. 11)

4 En el extremo opuesto se encuentran Colima, Nayarit, Nuevo León, Sinaloa y Sonora con tasas en torno a 40\%; mención aparte merece el Distrito Federal al contar con una tasa de más de $70 \%$. Sin duda la cobertura alcanzada en materia de educación superior es todavía muy insatisfactoria en el contraste internacional (UNAM, 2012). 
Para poder contribuir al referido desarrollo tanto de la Riviera Maya como de la entidad, es necesario que la UAPC proponga soluciones innovadoras y viables a las problemáticas del entorno. Esto no será posible si la Unidad no se sitúa en el mismo contexto que la comunidad en la que está insertada; si no colabora con la creación de un pensamiento capaz de ayudar en la construcción de una sociedad más justa, y si no refuerza su compromiso social (Marcuzzo, 1996, citado en Enríquez, 2006). Para ello, como señala Attali (1998, en Enríquez, 2006), se requiere que el estudiantado egresado haya aprendido al menos cuatro cosas: a ser ciudadanos y ciudadanas, a comunicar, a crear y a pensar críticamente. Sin duda el desarrollo de las habilidades o competencias enunciadas puede potenciarse significativamente a través del aprendizaje basado en la investigación (ABI) desde los primeros semestres en cada uno de los programas educativos ( $\mathrm{PE}^{\prime} \mathrm{s}$ ) que se imparten en la Unidad. Situación que representa un gran reto para la UAPC, dada la insuficiencia de su planta académica, aunada a otras condiciones relacionadas con la consolidación de la infraestructura física y administrativa, debido a su reciente creación.

Abordar cómo la articulación de la docencia con la investigación puede ser un factor para el fortalecimiento de los PE's de la Unidad es el objetivo del presente trabajo. En un primer apartado se describen, de manera general, las áreas de oportunidad que se tienen en la Unidad con base en el diagnóstico institucional y las recomendaciones realizadas por los Comités Interinstitucionales de Evaluación de la Educación Superior (CIEES) a los programas educativos que ya fueron evaluados; en un segundo apartado se realiza un acercamiento teórico al aprendizaje basado en la investigación (ABI) y a la investigación-acción como una herramienta metodológica apropiada tanto para la formación de jóvenes que investiguen como para el fortalecimiento de la planta docente. Posteriormente se presentan los resultados de la encuesta aplicada a 26 docentes de la UAPC -10 docentes de tiempo completo y 16 docentes de asignatura-respecto de los obstáculos, requerimientos e interés por aplicar el ABI en las aulas. Finalmente se presentan una serie de reflexiones finales. 


\section{Antecedentes: La investigación en la Unidad Académica Playa del Carmen}

Diferentes diagnósticos señalan la necesidad de fortalecer la investigación en la UAPC. El primero de ellos es el que se desprende del Plan Estratégico de Desarrollo Institucional de la Universidad (PEDI), el segundo es resultado de las observaciones recibidas por los Comités Interinstitucionales de Evaluación de la Educación Superior (CIEES). A continuación se analiza cada uno de ellos:

a) Diagnóstico del Plan Estratégico de Desarrollo Institucional de la Universidad (PEDI):

De la matriz FODA del PEDI, se extrajeron aquellos indicadores que tienen relación con la investigación a nivel institucional y se compararon con los datos de la Unidad Académica Playa del Carmen (UAPC). En general en la Tabla 1 se puede observar que las condiciones en cuanto a la habilitación del profesorado para la investigación son similares cuando el análisis se realiza exclusivamente con profesorado investigador de Carrera (PIC), a excepción de la tasa de profesorado incorporado al Sistema Nacional de Investigadores que es menor en la UAPC en comparación con la tasa institucional. Los números y las condiciones se manifiestan menos favorables cuando se consideran a todo el profesorado de tiempo completo de la UAPC (incluyendo a personal docente de carrera, extraordinario o auxiliar). No es de extrañar, entonces, que los indicadores de la UAPC sean más bajos comparados con los institucionales y la articulación entre la investigación y la docencia sea prácticamente nula. Asimismo destaca la escasa vinculación con redes de colaboración académica y la poca incidencia de la UAPC en la promoción, investigación, y difusión de la protección a los ecosistemas y el desarrollo sustentable en el municipio y la región. 


\section{Tabla 1}

\section{Comparativo institucional - UAPC de indicadores de habilitación del profesorado e impacto local en materia de investigación}

\begin{tabular}{|c|c|}
\hline Indicadores UQROO & Indicadores UAPC \\
\hline $\begin{array}{l}\text { El porcentaje de profesorado- } \\
\text { investigador de carrera (PIC) que cuenta } \\
\text { con estudios de posgrado (96\%) está por } \\
\text { encima de la media nacional ( } 86 \%) \text {. }\end{array}$ & $\begin{array}{l}\text { El } 72 \% \text { de los PTC tienen estudios de } \\
\text { posgrado (titulado). (14 de 18). } \\
100 \% \text { de PIC tienen estudios de posgrado } \\
\text { (titulados). ( } 8 \text { de } 8) .\end{array}$ \\
\hline $\begin{array}{l}\text { El porcentaje de PIC que cuenta con } \\
\text { grado de doctorado ( } 48 \% \text { ) está por } \\
\text { encima de la media nacional ( } 34 \%) \text {. }\end{array}$ & $\begin{array}{l}\text { El } 22 \% \text { de los PTC cuentan con el grado } \\
\text { de doctorado. ( } 4 \text { de } 18) \\
50 \% \text { de PIC cuenta con el grado de } \\
\text { doctorado }(4 \text { de } 8) .\end{array}$ \\
\hline $\begin{array}{l}\text { El porcentaje de PIC que cuenta con el } \\
\text { reconocimiento al Perfil deseable }(77 \%) \\
\text { de la Secretaría de Educación Pública } \\
\text { (Prodep) está por encima de la media } \\
\text { nacional }(43 \%) .\end{array}$ & $\begin{array}{l}28 \% \text { de PTC cuenta con perfil deseable } \\
(5 \text { de } 18) . \\
63 \% \text { de PIC cuenta con perfil deseable } \\
(5 \text { de } 8) .\end{array}$ \\
\hline $\begin{array}{l}\text { El porcentaje de PIC que cuenta con } \\
\text { el reconocimiento como profesorado } \\
\text { investigador nacional ( } 47 \%) \text { del Sistema } \\
\text { Nacional de Investigadores (SNI) } \\
\text { del Consejo Nacional de Ciencia y } \\
\text { Tecnología está por encima de la media } \\
\text { nacional }(17 \%) \text {. }\end{array}$ & $\begin{array}{l}\text { 17\% de PTC tiene reconocimiento del } \\
\text { SNI ( } 3 \text { de } 18) \\
38 \% \text { de PIC tiene reconocimiento del } \\
\text { SNI ( } 3 \text { de } 8 \text { ) }\end{array}$ \\
\hline $\begin{array}{l}\text { La institución cuenta con una } \\
\text { Comisión Institucional de Evaluación y } \\
\text { Seguimiento de los Cuerpos Académicos } \\
\text { (CIESCA) que funciona adecuadamente } \\
\text { y ha permitido mejorar su gestión. }\end{array}$ & $\begin{array}{l}\text { La UAPC no cuenta con una comisión } \\
\text { interna de evaluadora para la } \\
\text { investigación. Cuenta con un CA y un } \\
\text { GI. }\end{array}$ \\
\hline $\begin{array}{l}\text { Los PIC participan activamente en redes } \\
\text { de colaboración con otras instituciones } \\
\text { nacionales e internacionales. }\end{array}$ & $\begin{array}{l}\text { Los PTC tienen poca participación } \\
\text { en redes de colaboración nacionales e } \\
\text { internacionales. }\end{array}$ \\
\hline $\begin{array}{l}\text { La universidad tiene un papel } \\
\text { preponderante en la entidad como } \\
\text { promotor, investigador y divulgador } \\
\text { de la protección a los ecosistemas y el } \\
\text { desarrollo sustentable. }\end{array}$ & $\begin{array}{l}\text { Nulo papel como promotora, } \\
\text { investigadora y divulgadora de la } \\
\text { protección a los ecosistemas y el } \\
\text { desarrollo sustentable. }\end{array}$ \\
\hline
\end{tabular}

Nota: Elaboración propia con base en el Plan Estratégico de Desarrollo Institucional (PEDI) 2013-2016 (UQROO, 2013-2016).

De lo anterior se desprende, por un lado, la necesidad de fortalecer las capacidades investigativas del profesorado que actualmente no 
cuenta con perfil del Programa para el Desarrollo Profesional Docente, para el tipo superior (PRODEP) o Sistema Nacional de Investigadores (SIN) y su articulación con la docencia y, por el otro, elevar los perfiles de las nuevas contrataciones de profesorado, que deben solicitar experiencia de investigación probada, dado que la calidad educativa de una institución de educación superior (IES) está determinada fundamentalmente por la calidad de su planta académica.

Finalmente, en el PEDI se reconoce la necesidad de contar con una mayor integración entre los sistemas de información universitaria que permita generar datos para la toma de decisiones a nivel institucional (UQROO, 2013-2016).

\section{b) Evaluaciones diagnósticas de los Comités de Evaluación de la Edu- cación Superior (CIEES):}

En los años 2014 y 2015 se realizaron las evaluaciones de los Comités de Evaluación de la Educación Superior (CIEES) para los programas educativos (PE) de Administración Hotelera (AH), Ingeniería Empresarial (IEM) y Gobierno y Gestión Pública (GyGP), mediante las respectivas visitas prediagnósticas y diagnósticas a cada PE mencionado. Si bien es cierto que existe una diferencia en cuanto a los términos de las recomendaciones -más favorables para GYGP-, en general, es notoria la insuficiencia en las actividades de investigación y, sobre todo, su vinculación con la docencia.

Enla mayoría, los informes elaborados por los distintos comités CIEES que visitaron la Unidad para evaluar los programas educativos referidos, mencionan que para las tres carreras existe un desequilibrio en las actividades de docencia, gestión, tutoría e investigación. De ahí las recomendaciones de los CIEES de contratar más profesorado de tiempo completo por concursos de oposición, crear cuerpos académicos relacionados con los programas educativos o consolidar al grupo y cuerpo académico existente mediante la apertura o ampliación de líneas de investigación relacionadas con los programas educativos que permitan involucrar al estudiantado en los nuevos proyectos de investigación. Como se muestra en la Tabla 2 a manera de resumen. 


\section{Tabla 2}

\section{Concentrado de recomendaciones de los CIEES para las tres carreras evaluadas}

\begin{tabular}{|c|c|}
\hline Programa educativo (PE) & $\begin{array}{c}\text { Recomendaciones de los CIEES en cuanto a } \\
\text { la investigación }\end{array}$ \\
\hline \multirow[t]{3}{*}{$\begin{array}{l}\text { Administración Hotelera } \\
(\mathrm{AH})\end{array}$} & $\begin{array}{l}\text { "Formar un cuerpo académico de la } \\
\text { disciplina" }\end{array}$ \\
\hline & $\begin{array}{l}\text { "Incrementar la investigación que apoye } \\
\text { la línea de generación y aplicación del } \\
\text { conocimiento del PE" }\end{array}$ \\
\hline & $\begin{array}{l}\text { "Fortalecer la articulación entre la } \\
\text { investigación y la docencia" } \\
\end{array}$ \\
\hline \multirow[t]{3}{*}{$\begin{array}{l}\text { Ingeniería Empresarial } \\
\text { (IEM) }\end{array}$} & $\begin{array}{l}\text { "Formar un cuerpo académico reconocido } \\
\text { por el PRODEP" }\end{array}$ \\
\hline & "Incrementar la producción académica" \\
\hline & $\begin{array}{l}\text { "Desarrollar la función sustantiva de } \\
\text { investigación" }\end{array}$ \\
\hline \multirow{5}{*}{$\begin{array}{l}\text { Gobierno y Gestión Pública } \\
\text { (GyGP) }\end{array}$} & "Promover facilidades para la investigación" \\
\hline & $\begin{array}{l}\text { "Consolidar el cuerpo académico existente y } \\
\text { establecer un cuerpo académico más" }\end{array}$ \\
\hline & $\begin{array}{l}\text { "Garantizar condiciones para que los } \\
\text { profesores investigadores continúen como } \\
\text { miembros del SNI y el PRODEP" }\end{array}$ \\
\hline & $\begin{array}{l}\text { "Otorgar facilidades para que los profesores } \\
\text { participen en eventos académicos" }\end{array}$ \\
\hline & $\begin{array}{l}\text { "Ampliar las líneas de investigación y } \\
\text { aplicación del conocimiento del cuerpo } \\
\text { académico" }\end{array}$ \\
\hline
\end{tabular}

Nota: Elaboración propia con base en los Informes CIEES (2014a, 2014b, 2015a y 2015b).

Por tanto, resulta evidente que para asegurar la calidad educativa y fortalecer la producción de conocimiento, es primordial pensar en estrategias que vinculen la investigación con la docencia como un primer paso para analizar de qué manera se puede lograr la articulación referida. A continuación se realiza un acercamiento teórico al aprendizaje basado en la investigación como un posible camino a seguir en la UAPC. 


\section{Marco teórico: Un acercamiento teórico al aprendizaje basado en la investigación (ABI)}

Diversas investigaciones (Buendía y Salmerón, 1994; Elliot, 1981; Lapuebla, 1988; Lewin, 1973; López-Górriz, 1993) han destacado la importancia de la vinculación entre la docencia y la investigación. En general, estas refieren la necesidad de transformar los procesos de aprendizaje de tal forma que las alumnas y los alumnos sean sujetos activos de su propia formación. Otras, como las de Griffiths (2004) y Healey y Jenkis (2009) desarrollaron un marco teórico que ayuda a conceptualizar cómo se puede vincular la investigación con el entorno de aprendizaje de estudiantes de licenciatura, dependiendo de si el aprendizaje está centrado en estudiantes o en el docentes, y si el aprendizaje se centra en el producto de la investigación o en el proceso de investigación (Peñaherrera, Chiluiza y Ortiz, 2014). Por ejemplo, en la enseñanza orientada a la investigación (Research Oriented Teaching), la figura predominante es el personal docente y el estudiantado aprende acerca de los procesos de investigación, cómo se crea el conocimiento y cómo funciona la mente de la persona investigadora; mientras que en el aprendizaje basado en la investigación (ABI) -en inglés llamado Research Based Learning (RBL)- el estudiantado actúa como investigador, aprende habilidades asociadas y realiza indagaciones e investigaciones de corto alcance (Peñaherrera et al., 2014, p.208).

El aprendizaje basado en investigación (ABI) es un modelo que surge a partir de las críticas realizadas por Ernest Boyer (educador norteamericano) a fines de la década de los años 90 del siglo pasado, sobre la falta de participación del estudiantado en actividades de investigación y la consecuente carencia de habilidades relacionadas con esta misma (Martínez y Buendía, 2005; Torres, 2012, como se cita en Peñaherrera et al., 2014). Como bien señalan Peñaherrera et al. (2014), la alfabetización científica o el desarrollo de la "capacidad de investigación" ${ }^{\prime \prime}$ es indispensable dados los retos que se tienen hoy en día a nivel global y local:

5 Entendida como "aquellas habilidades que les permiten [al alumnado] hacer frente a nuevas situaciones, utilizar el pensamiento de orden superior en situaciones más abiertas y cambiantes, vinculando todo a un proceso creativo, generativo y reflexivo" (Roach, Blackmore y Dempster, 2001 citado en Peñaherrera et al., 2014, p. 210). 
Cada vez más es evidente la necesidad de profesionales que sean capaces, no solo de replicar conocimiento, sino de crear: conocimientos, tecnologías, formas de analizar información, entre otros. El ABI propone desarrollar este tipo de habilidades en los estudiantes, siempre y cuando de manera efectiva los docentes las promuevan y las hagan realidad en el aula y en las actividades que se proponen... . El modelo de aprendizaje basado en la investigación es un modelo coherente con la didáctica actual, que se basa en la idea de que los estudiantes se apropien y construyan conocimientos cimentados en la experiencia práctica, el trabajo autónomo, el aprendizaje colaborativo y por descubrimiento, rubros fundamentales para alcanzar dominios en los aprendizajes, desarrollar conocimientos y actitudes para la innovación científica, tecnológica, humanística y social. (2014, pp. 206-207)

De hecho, el ABI, como estrategia didáctica y como una de las referencias más frecuentes cuando se habla de innovación educativa, cobra cada vez más fuerza en diferentes latitudes como ejemplo de un paradigma que fomenta el aprendizaje significativo ${ }^{6}$, dado que presenta diversas ventajas ${ }^{7}$ que contribuyen a la formación profesional, al

6 La Universidad de Warwick en el Reino Unido desarrolló el modelo ABI en las diferentes titulaciones de pregrado; el South Carolina Honors College utiliza la investigación como una estrategia de tipo curricular que permite a su estudiantado egresado ser más competitivo para conseguir becas y ser admitido en escuelas profesionales (Martínez y Buendía, 2005 en Peñaherrera et al., 2014); el Consejo de Educación Superior de Ecuador, aprobó en 2013 el Reglamento de Régimen (RRA) que establece como política educativa que la investigación forme parte de la formación académica y profesional, y que esta se vincule con la sociedad en un marco de calidad, innovación y pertinencia (RRA, Título 1, art. 2 literal a y d en Peñaherrera et al., 2014). Asimismo, la Comisión Boyer para la Educación de las Universidades de Investigación en Estados Unidos recomendaba el ABI; por otro lado, en 2013 el Consejo de Investigación de Pregrado en los Estados Unidos (US Council on Undergraduate Research), también resalta la importancia de la integración de la investigación y la docencia al definir a la primera como aquella llevada a cabo por estudiantado universitario que hace una contribución intelectual, creativa y original a la disciplina. Finalmente, la Unión Europea reconoció al aprendizaje basado en la investigación / indagación (Research or Inquiry Based Learning) como la metodología idónea para la enseñanza de las ciencias y las matemáticas (Peñaherrera et al., 2014).

7 Presenta las siguientes ventajas: Introduce a estudiantes en el camino de la investigación y fortalece al profesorado; establece un vínculo entre los cursos académicos y las áreas potenciales de investigación de la institución y grupos de investigación; promueve que el alumnado sea capaz de desarrollar las habilidades y competencias necesarias para investigar -tales como pensamiento crítico, análisis, síntesis, liderazgo, creatividad, emprendimiento, resolución de problemas, entre otras-, con la finalidad de involucrarlo en el proceso de descubrimiento científico dentro del trabajo en el aula en sus respectivas disciplinas; el estudiantado aprende en el contexto de la 
generar actitudes de crítica y renovación, lo que, sin duda, favorece el cambio y la transformación del entorno a través de la participación; refuerzan la concientización de los sujetos en el desarrollo social; además de transformar actitudes y comportamientos que posibilitan el desarrollo personal (Bausela, 2004).

Pocos son los casos documentados en el contexto latinoamericano en materia de articulación de la investigación con la docencia, sobresale el Tecnológico de Monterrey -una de las principales universidades privadas del país- que concibe al $\mathrm{ABI}$ como:

La aplicación de estrategias de enseñanza y aprendizaje que tienen como propósito conectar la investigación con la enseñanza, las cuales permiten la incorporación parcial o total del estudiante en una investigación basada en métodos científicos, bajo la supervisión del profesor. (ITESM, 2010 como se cita en Peñaherrera et al., 2014, p. 208)

Y la ha convertido en la estrategia para desarrollar una cultura de investigación tanto en estudiantes como en docentes, pues propone lo siguiente:

Que el aprendizaje se construya en escenarios reales que vinculen a estudiantes y profesores en un proceso de reconstrucción de conocimientos inspirados en los principios de la investigación científica... . No se trata de pensar a la investigación desde la investigación, sino pensarla desde la labor didáctica y pedagógica. (Peñaherrera et al., 2014, p. 208)

investigación, busca nuevos conocimientos y adquiere compromisos con el aprendizaje permanente; promueve la interacción entre la enseñanza y la investigación, y estos con estudiantes; el personal docente tiene la posibilidad de orientar todo el proceso de investigación de forma más eficiente, en la medida en que las experiencias exitosas pueden extrapolarse en el aula; apoya el aumento de la confianza y habilidades transversales como la resolución de problemas y el desarrollo del pensamiento crítico de estudiantes; estimula el desarrollo cognitivo y habilidad técnica de los sujetos participantes; ayuda al alumnado a tener mayor claridad sobre su futuro profesional o de las oportunidades de educación y al profesorado a tener una mejor comprensión de cómo se crea el conocimiento, así como las formas disciplinarias de pensar y practicar. Estas ventajas se generaron luego de hacer la revisión de las ideas de varios textos (Chávez, 2013; Gollete y Lesgard-Hervert, 1988, como se cita en Bausela, 2004; Hunter, Laursen y Seymour et al., 2007; Hunter, Laursen, Seymour, Thiry y Melton, 2010; Morales, Rincón y Romero, 2004; Rojas, 2009; Torres, 2012, en Peñaherrera et al., 2014). 
Otro caso a destacar es la experiencia de la Facultad de Medicina de la UNAM, en donde a partir de la incorporación temprana de estudiantes a la investigación por medio del Programa de Apoyo y Fomento a la Investigación Estudiantil (AFINES), el cual tiene como objetivo preparar el alumnado para realizar proyectos de investigación, un grupo de estudiantes sobresalientes desarrollaron protocolos de investigación y lograron publicar sus resultados. Como parte del Programa se llevan expedientes del alumnado participante que contienen copias de sus proyectos, comunicaciones de congresos, artículos de difusión y artículos de investigación (Rodríguez-Paz y Gijón, 2000).

Una de las modalidades de la ABI es la investigación-acción; de hecho, si bien es cierto que este paradigma surgió desde mediados del siglo pasado como una auténtica metodología de práctica reflexiva sobre el quehacer docente, hoy más que nunca cobra vigencia como un mecanismo fundamental para la articulación de la docencia y la investigación desde el aula. ${ }^{8}$ Según Contreras (1994), entre los hitos más significativos en la historia de la investigación-acción, se encuentran, en primer lugar los trabajos de Kurt Lewin $(1946,1952)$ en los Estados Unidos de Norteamérica, en donde el autor expresa que es importante la producción académica (libros, artículos); pero que también es necesario integrar la investigación científica con la acción social. Lewin definió la investigación-acción "como un proceso cíclico de exploración, actuación y valoración de resultados" (como se cita en Bausela, 2004, p. 1), y la concibió como una investigación "emprendida por personas, grupos o comunidades que llevan a cabo una actividad colectiva en bien de todos, consistente en una práctica reflexiva social en la que interactúan la teoría y la práctica con miras a establecer cambios apropiados en la situación estudiada y en la que no hay distinción entre lo que se investiga, quién investiga y el proceso de investigación" (Restrepo, 2005, p. 159 en Colmenares y Piñero, 2008, p. 98). En segundo lugar, en los años setenta del siglo XX en la Gran Bretaña, para Lawrence Stenhouse y John Elliot la investigación-acción refiere a lo siguiente:

8 Sobre el particular, Colmenares y Piñero consideran a la IA como una "herramienta metodológica para estudiar la realidad educativa, mejorar su comprensión, y al mismo tiempo logar su transformación .... Por ello se plantea la necesidad de asumir una concepción socioconstructivista de la realidad social, en la cual se generan espacio por y entre los actores sociales para el diálogo, la reflexión y la co-construcción del conocimiento sobre los diferentes problemas que puedan afectar los actos y prácticas educativas dentro y fuera del área" (2008, p. 96). 
Ya no significa solamente una técnica de investigación para ocasionar cambios, sino la convicción de que las ideas educativas sólo pueden expresar su auténtico valor cuando se intenta traducirlas a la práctica, y esto sólo pueden hacerlo los enseñantes investigando con su práctica y con las ideas con las que intentan guiarse. (Stenhouse, 1984, como se cita en Bausela, 2004, p. 2)

Finalmente, en Australia, en los años ochenta del siglo pasado, Stephen Kemis junto con Wilfred Carr y el equipo de la Universidad de Deakin intentaron una nueva definición y determinaron que "la [investigación-acción] no puede entenderse como un proceso de transformación de las prácticas individuales del profesorado, sino como un proceso de cambio social que se emprende colectivamente" (Bausela, 2004, p. 2). En general, los hitos referidos ejemplifican dos grandes tendencias, una netamente sociológica cuyo punto de partida fueron los trabajos de Kurt Lewin, que fueron continuados por el antropólogo norteamericano Sol Tax (1958) y el sociólogo colombiano Fals Borda (1970), quien les imprimió una marcada connotación ideológica y política; mientras que la segunda vertiente es más educativa y está inspirada en las ideas de Paulo Freire (1974) en Brasil, Stenhouse (1984) y su discípulo John Elliot (1981) en Inglaterra, así como por Carr y Kemmis (1988) en la referida Universidad de Deakin en Australia (Colmenares y Piñero, 2008).

Así, pues, la investigación-acción se presenta como una metodología de investigación que busca el cambio educativo y la transformación social, y se caracteriza entre otras cuestiones por las siguientes:

(i) Se construye desde y para la práctica, (ii) pretende mejorar la práctica a través de su trasformación, al mismo tiempo que procura comprenderla, (iii) demanda la participación de los sujetos en la mejora de sus propias prácticas, (iv) exige una actuación grupal por la que los sujetos implicados colaboran coordinadamente en todas las fases del proceso de investigación, (v) implica la realización de análisis crítico de las situaciones y (vi) se configura como una espiral de ciclos de planificación, acción, observación y reflexión. (Kemmis y MacTaggart, 1988 como se cita en Bausela, 2004, p. 3) 
A las cuestiones anteriores hay que añadir que la investigación- acción necesita de condiciones laborales que la hagan posible, se requiere de tiempo para la discusión con colegas, la planificación conjunta, la recolección de datos ${ }^{9}$, la sistematización y el análisis; no se lleva a cabo exclusivamente en el aula y no puede ser nunca una tarea individual -debe ser, por el contrario, un trabajo cooperativo-, dado que cualquier tarea de investigación requiere un contexto social de intercambio, discusión y contrastación (Bausela, 2004). Así pues, la investigación-acción "no se limita a someter a prueba determinadas hipótesis o a utilizar datos para llegar a conclusiones, ... es un proceso que sigue una evolución sistemática, y cambia tanto al investigador como a las situaciones en las que éste actúa" (Bausela, 2004, p. 3).

De forma general, la investigación-acción se desarrolla siguiendo un modelo en espiral en ciclos sucesivos que incluyen diagnóstico, planificación, acción, observación y reflexión-evaluación. Todo este proceso se resume en cuatro fases: 1) Diagnóstico y reconocimiento de la situación inicial; 2) Desarrollo de un plan de acción, críticamente informado, para mejorar aquello que ya está ocurriendo; 3) Actuación para poner el plan en práctica y la observación de sus efectos en el contexto que tiene lugar, y 4) La reflexión en torno a los efectos como base para una nueva planificación (Bausela, 2004).

Cabe destacar que el modelo referido no es el único y que de hecho puede configurarse un modelo propio por los actores sociales involucrados en él. Sin embargo, como señalan Colmenares y Piñero:

... cualquier modelo a seguir debe partir del diagnóstico de una situación problema, cuya solución resulta de la planificación, ejecución y evaluación de acciones conjuntas. Otro aspecto interesante, es que tanto la reflexión, como la recogida y sistematización de la información se constituyen en los ejes transversales que permean todo el desarrollo del proceso; es por ello que el docente investigador o grupo investigador, debe generar los espacios para la discusión y la valoración de los logros y limitaciones

9 La información necesaria para el diagnóstico y reconocimiento de la situación inicial se obtiene a partir de estudios cuantitativos, observaciones y los diarios de campo, además del análisis de documentos, datos fotográficos, grabaciones en audio y video, entrevistas y encuestas de opinión, registros anecdóticos, estudios de caso, grupos focales, círculos de reflexión, entre otros (Bausela, 2004, p. 7; Colmenares y Piñero, 2008, pp.106-107). 
hasta el momento alcanzados, con el fin de tomar decisiones sobre el rumbo a seguir, y sobre la actuación de cada uno de los actores, en forma colectiva e individual. Igualmente, la información recolectada debe ser debidamente registrada, sistematizada y contrastada, utilizando para ello las técnicas de triangulación, tales como la triangulación de perspectivas o datos, triangulación metodológica, triangulación de investigadores, y bien pudiera utilizarse también la triangulación teórica. Una vez culminada la investigación se procede a escribir el informe final, el cual debe ser de carácter descriptivo, haciendo uso de un lenguaje sencillo sin que ello signifique disminuir el rigor y la seriedad del análisis, en dicho escrito es importante agregar sentimientos, actitudes y percepciones de los implicados. Y finalmente, los resultados o hallazgos encontrados deben hacerse públicos entre la comunidad de actores sociales implicados, y por qué no, divulgarlos en forma escrita u oral, a través de la presentación de trabajos investigativos, artículos en revistas especializadas, ponencias en eventos vinculados con la temática, entre otros. (2008, pp. 108-109)

Conviene preguntarse, entonces, qué tipo de aprendizaje basado en la investigación se necesita implementar en la UAPC. Nos queda claro que uno que fomente decididamente la articulación de la docencia con una investigación que responda a la misión de la Unidad, de la cual se desprendan las diversas líneas de generación y aplicación del conocimiento (LGAC) para cada programa educativo, las que deberían ser la base para organizar grupos de investigación y cuerpos académicos. Nos queda claro también que la investigación-acción es una metodología adecuada para este tipo de aprendizaje, dado que permite una vinculación con los problemas del entorno, pero sobre se intenta incidir en la solución o disminución de estos mismos.

En el siguiente apartado se mostrarán los resultados de la encuesta realizada a docentes de la Unidad con la finalidad de conocer la disposición y los obstáculos para implementar la ABI.

\section{Metodología}

Para abordar la presente investigación se formuló una pregunta de investigación que derivó de la revisión teórica: ¿Cómo se pueden 
fortalecer los programas educativos en educación superior por medio de articulación de la docencia con la investigación? En concordancia con la naturaleza de esta interrogante, se eligió utilizar la metodología mixta, por ser apropiada para obtener este tipo de información y posteriormente poder analizare interpretar su significado, a partir de la investigación y de reflexiones personales (Creswell, 2012).

Para ello se siguieron los siguientes pasos: se identificó el contexto y las fuentes de información; se determinaron los datos a obtener; se elaboraron los instrumentos y se realizó una prueba piloto para verificar su funcionamiento. En una primera etapa se llevó a cabo la revisión de documentos significativos y la bibliografia especializada como técnicas cualitativas iniciales, con base en el análisis de la información obtenida se diseñó un cuestionario estructurado para llevar a cabo una encuesta en la parte cuantitativa. Dicha encuesta que contenía tres preguntas -dos de ellas, con siete y 15 reactivos respectivamente, fueron medidas con la escala de Likert, mientras que la última pregunta fue abierta- se desarrolló en el programa Surveymonkey y se envió el 27 de junio del 2016 por correo electrónico a un total de 92 docentes de las cuatro carreras, de quienes respondió el 28\%. Una vez recogida la información, se trianguló con el marco teórico para poder hacer un análisis a profundidad y poder con ello conocer la disposición y los obstáculos para implementar el ABI.

De esta forma, se pudieron validar los datos y se aseguró la confiabilidad de las conclusiones. Asimismo, se dio sentido a la evidencia por medio de que se confrontaron los datos emanados de distintas fuentes de información con diferentes instrumentos de recolección (Creswell y Plano-Clark, 2011). A continuación se presentan los resultados obtenidos.

\section{Resultados y discusión}

Como se señaló en la introducción, la encuesta se envió por el correo institucional a todo el profesorado, el $28 \%$ respondió. El instrumento se diseñó para determinar el interés delpersonal docente en el ABI y los obstáculos que detecta para su implementación en el salón de clases. Para efectos comparativos, las respuestas se desagregaron por tipo de docente (de tiempo completo y de asignatura). En la Figura 1 se muestran el grado de aceptación o rechazo a diferentes postulados relacionados con los impedimentos o causas que pueden obstaculizar la implementación del ABI 
en el salón de clases. Para ello se utilizó la escala de Likert -en donde 1 equivale a totalmente en desacuerdo; 2 en desacuerdo; 3 ni de acuerdo ni en desacuerdo; 4 de acuerdo y 5 totalmente de acuerdo. Lo primero que se advierte en la gráfica es que una buena parte del profesorado de tiempo completo rechaza en mayor medida que el de asignatura, desconocer las competencias de egreso de cada PE o los problemas locales; reconoce también que pueden relacionar la materia con el contexto local. Resalta también que independientemente del tipo de docente no se está ni de acuerdo, ni en desacuerdo con los siguientes supuestos: "el estudiantado desconoce la utilidad de la investigación" y "el estudiantado no cuenta con bases de investigación", situación que refleja que se ha reflexionado poco o más bien nada sobre el tema, y que en general el cuerpo docente no ve al alumnado como investigador potencial, ¿será esto un reflejo de una concepción de la investigación como una tarea muy compleja o solo para las élites académicas o estudiantes de posgrado? Habrá que explorar esto con mayor profundidad. Otro indicador que demuestra nuevamente la necesidad de seguir trabajando en el fortalecimiento de las capacidades investigativas de docentes es el propio reconocimiento de una parte del profesorado de tiempo completo de que no tienen la suficiente experiencia en investigación, y de que tampoco tienen los conocimientos teóricos sobre el ABI; situación que contrasta con el desacuerdo de docentes de asignatura a los siguientes postulados "no tengo suficiente experiencia en investigación", "no tengo los conocimientos teóricos". 


\section{Figura 1}

Causas o barreras para no utilizar el ABI en el salón de clases. Elaboración propia con base en las encuestas aplicadas en junio de 2016

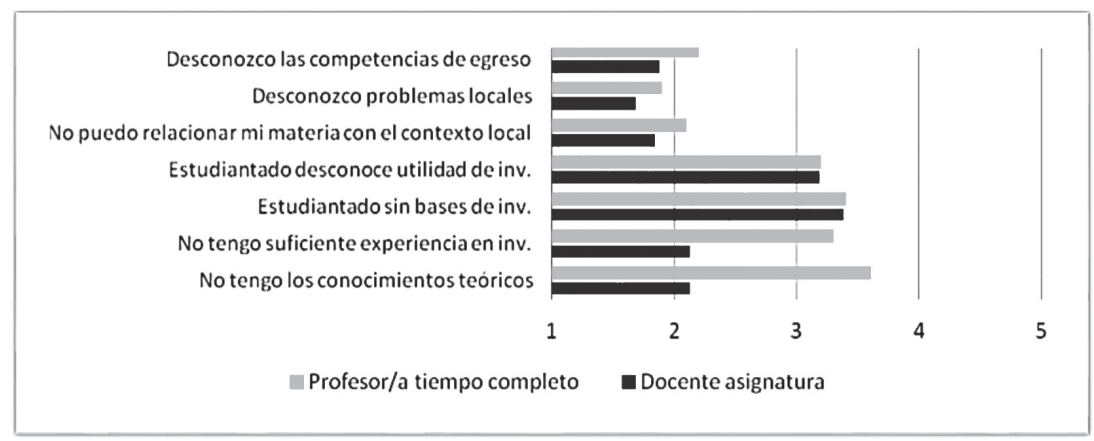

La segunda parte de la encuesta está conformada por 15 reactivos cuyo análisis se presenta en las siguientes tres gráficas. En la Figura 2 se señala el grado de aceptación del profesorado respecto de los temas que requieren conocer para utilizar el $\mathrm{ABI}$ en el aula. Resalta el hecho de que docentes de tiempo completo refieran en mayor grado que el profesorado de asignatura que requieren cursos sobre escritura científica y ABI. Igualmente importante y pertinente le parece a todo el profesorado conocer el perfil socioeconómico de sus estudiantes. Por último, la mayoría no está seguro de conocer con profundidad el contexto local, dado que seleccionaron la opción 3 (ni de acuerdo ni en desacuerdo). 


\section{Figura 2}

Capacitación requerida por la planta docente. Elaboración propia con base en las encuestas aplicadas en junio de 2016

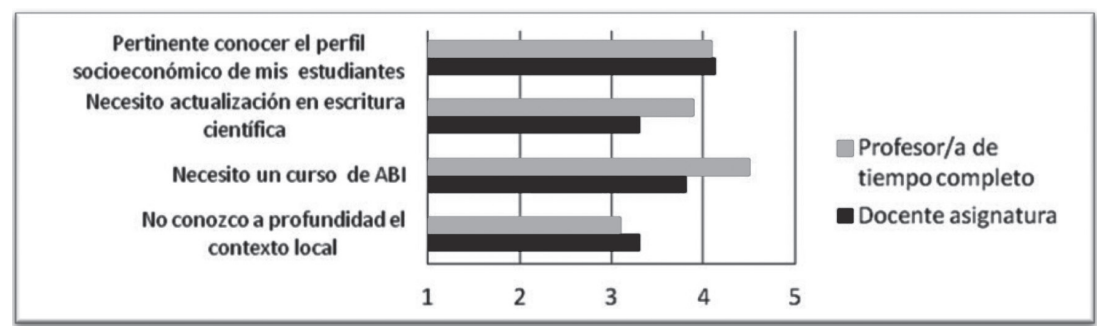

El siguiente grupo de reactivos se presenta en la Figura 3 y está relacionado con el interés de docentes de asignatura y de tiempo completo en la investigación educativa. Lo primero que destaca es que el profesorado de tiempo completo está de acuerdo en mayor grado (escala 4.5 de 5) en comparación con el de asignatura, en que la investigación facilita la innovación educativa y el desarrollo profesional, y permite reflexionar sobre el propio quehacer docente (escala 4.1 de 5). En segundo lugar se advierte que el profesorado de asignatura está más interesado en realizar investigación educativa en sus grupos (3.9 vs. 3.7 que el de tiempo completo). Finalmente, de manera general todo el profesorado está de acuerdo en formar parte de un grupo de docentes que realiza investigación educativa.

\section{Figura 3}

Interés del profesorado en la investigación educativa. Elaboración propia con base en las encuestas aplicadas en junio de 2016

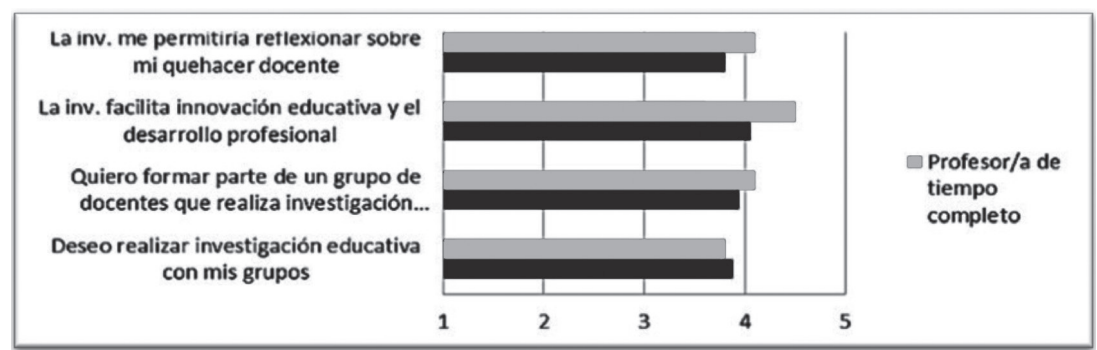


El último grupo de reactivos analizados se presenta en la Figura 4 y está relacionado con el desarrollo de acciones para potenciar la investigación colectiva entre estudiantes y docentes. En general el personal docente está de acuerdo o casi totalmente de acuerdo en que es pertinente incluir al alumnado en las investigaciones, en la conveniencia de vincular las asignaturas con proyectos de investigación y en implementar un programa de apoyo y formación de investigación para estudiantes. Asimismo, se observa que el cuerpo docente están casi totalmente de acuerdo en vincular las asignaturas con proyectos de investigación que involucren varias materias, y en apoyar a docentes de asignatura con un programa de fomento de investigación. Este último grupo manifestó estar de acuerdo con los postulados; pero en un grado menor al profesorado de tiempo completo.

\section{Figura 4}

\section{Interés en la investigación colectiva. Elaboración propia con base en las encuestas aplicadas en junio de 2016}

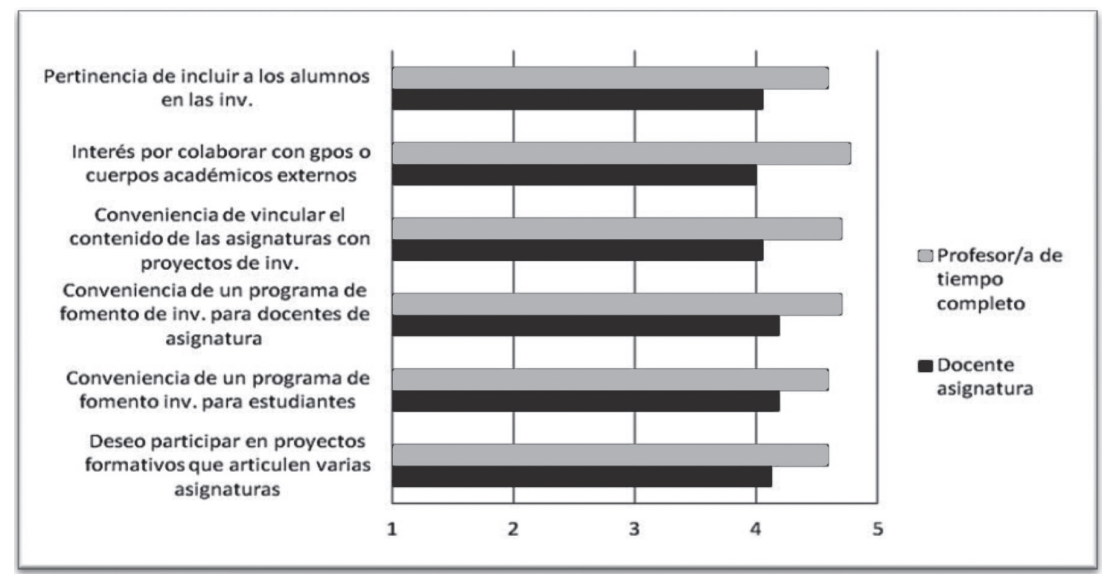

Finalmente, en la tercera sección de la encuesta se hizo una pregunta abierta para explorar posibles métodos de evaluación para el ABI en el salón de clases. Entre las respuestas más frecuentes sobresalen las siguientes: evaluación de pares, ensayo con metodología científica, artículo científico, ponencia, participación en foros, proyectos piloto puestos en práctica, reportes monográficos, evaluación de competencias, producto científico publicado, participación en proyectos de 
investigación, evaluaciones que incluyan conjuntamente el proceso, resultados, implementación y autoevaluación del desempeño; evaluaciones en tres momentos diagnóstica, formativa y final que en conjunto incluyan el proceso de aprendizaje, las competencias desarrolladas, el ensayo científico utilizando APA; referencias bibliográficas, categorías de análisis y conceptualizaciones.

\section{Conclusiones}

De acuerdo con Caballero y Bolívar (2015), las concepciones sobre la enseñanza y el aprendizaje dependen del nivel de experiencia que tenga el personal docente en un momento dado. En la etapa inicial (principiante), generalmente la preocupación docente gira en torno a sí mismo o misma y en dominar su materia; conforme adquiere mayor experiencia se preocupa por el aprendizaje del estudiantado y las nuevas estrategias que utiliza en el aula lo conciben como un sujeto activo, es hasta este momento cuando el docente tiene como objetivo formar un sujeto independiente. En este aprendizaje constructivista que se caracteriza fundamentalmente por buscar la autonomía del estudiantado -el cual es el ideal que cada docente debe perseguir- es donde precisamente la investigación puede jugar un papel preponderante.

Es pues, la docencia una disciplina que requiere de una formación teórico-práctica, adaptada a la particularidad de los contenidos que se quieren enseñar (Loughran, 2009, como se cita en Caballero et al., 2015). Es decir, no basta con tomar un curso de capacitación, sino que se requiere de un programa o plan estratégico que considere el cambio de concepciones, actitudes y procedimientos que se busca en el profesorado para lograr la vinculación de la docencia con la investigación que contribuya al aprendizaje significativo. Sin duda es un camino que la UAPC tendrá que recorrer para cumplir la misión que guía su quehacer.

Finalmente, es importante mencionar que adicionalmente al plan estratégico (o programa) mencionado, es necesario implementar políticas educativas institucionales (visibles en los apoyos e incentivos) que coordinen los procesos de selección, formación, evaluación y promoción del profesorado (De Miguel, 2003, como se cita en Caballero et $a l ., 2015)$ y que apoyen la integración de estas dos funciones sustantivas -la investigación y la docencia-. De esta forma, la colaboración entre personal investigador-personal docente y estuiantes aseguraría 
la inserción de estilos de aprendizaje transformadores que apoyen un genuino compromiso con el aprendizaje para toda la vida, además de constituir una herramienta educativa capaz de tratar las necesidades psicoeducativas requeridas para formar seres humanos autónomos en su formación e interesados en desarrollar competencias para el aprendizaje continuo. Solo así se estará en posibilidades de ofrecer al alumnado una educación de calidad, acorde con la realidad cambiante y altamente competitiva del siglo XXI.

\section{Referencias}

Bausela, E. (2004). La docencia a través de la investigación-acción. Revista Iberoamericana de Educación, 15, 1-9. Recuperado de rieoei.org/deloslectores/682Bausela.PDF

Buendía, L. y Salmerón, H. (1994). Intervención cooperativa a través de la investigación cooperativa. Revista de Investigación Educativa, 23, 226 - 231. Recuperado de http://www.redalyc.org/ pdf/175/17515081009.pdf

Caballero, K. yBolívar A. (2015). El profesorado universitario como docente: Hacia una identidad profesional que integre docencia e investigación. Revista de docencia universitaria,13(1), 57-77. Recuperado de https://dialnet.unirioja.es/servlet/ articulo? codigo $=5027836$

Carr, W. y Kemmis, S. (1988). Teoría crítica de la enseñanza. La investigación-acción en la formación del profesorado. Barcelona: Martínez Roca.

Chávez, G. (2013). La investigación formativa en la universidad. Proyecto de investigación del Cuerpo Académico "Cambio educativo: discursos, actores y prácticas”. Universidad Autónoma de Nuevo León, Nuevo León: México.

Colmenares, E. y Piñero, M. (2008). La investigación acción. Una herramienta metodológica heurística para la comprensión y transformación de realidades y prácticas socio-educativas. Laurus, 14(27) 96-114. Recuperado de http://www.redalyc.org/articulo. oa? id $=76111892006$

CIEES. (2014a). Informe de evaluación prediagnóstica. Licenciatura en Ingeniería Empresarial. Playa del Carmen: Comités Interinstitucionales para la Evaluación de la Educación Superior. 
CIEES. (2015a). Informe de evaluación. Licenciatura en Ingeniería Empresarial. Playa del Carmen: Comités Interinstitucionales para la Evaluación de la Educación Superior.

CIEES. (2014b). Informe de evaluación. Licenciatura en Administración Hotelera. Playa del Carmen: Comités Interinstitucionales para la Evaluación de la Educación Superior.

CIEES. (2015b). Informe de evaluación. Licenciatura en Gobierno y Gestión Pública. Playa del Carmen: Comités Interinstitucionales para la Evaluación de la Educación Superior.

Creswell, J. (2012). Educational research: Planning, conducting, and evaluating quantitative and qualitative research. Boston: Pearson. Recuperado de http://www.onlinecef.net/file.php/1/ CEF_Resources/Research\%20\%20Method/_Educational_Research_Planning__Conducting_and_Evaluating_Quantitative_and_Qualitative_Research_4th_Edition_.pdf

Creswell, J. W., Klassen, A. C., Plano Clark, V. L., \& Smith, K. C. (2011). Best practices for mixed methods research in the health sciences. Bethesda (Maryland): National Institutes of Health, 2094-2103.

Dempster, J. y Blackmore, P. (2002). Developing Research-Based Learning Using ICT in Higher Education Curricula: The Role of Research and Evaluation. En R. Macdonald \& J. Wisdom (Eds.), Academic and Educational Development: Research, Evaluation and Changing Practice in Higher Education (pp. 129-139). London: Kogan Page.

Elliot, J. (1981). The Teacher as a Researcher within awardbearing courses. En R. Alexander y J. Ellis (Eds.), Advanced Study for Teachers, SRHE. Nafferton Books Distributor.

Elliot, J. (2005). El cambio educativo desde la investigación acción. Madrid: Morata.

Enríquez Clavero, J. O. (2006). Educación superior: Tendencias y desafíos. Educación Médica, 9(1), 06-10. Recuperado de http://scielo.isciii.es/pdf/edu/v9n1/colaboracion2.pdf

Freire, P. (1974). Concientización. Teoría y práctica de la liberación. Buenos Aires: Búsqueda.

Griffiths, R. (2004). Knowledge Production and the Research-Learning Nexus: The Case of the Built Environment disciplines. Studies in Higher Education, 29(6), 709-726. 
Healey, M. y Jenkins, A. (2009). Undergraduate Research and Inquiry. New York: Higher Education Academy.

Hunter, A. B., Laursen, S. L., y Seymour, E. (2007). Becoming a Scientist: The Role of Undergraduate Research in Students' Cognitive, Personal, and Professional Development. Science Education, (91), 36-74.

Hunter A-B., Laursen S., Seymour E., Thiry H., y Melton G. (2010). Summer Scientists: Establishing the Value of Shared Research for Science Faculty and their Students. San Francisco: Jossey-Bass.

Instituto Tecnológico de Estudios Superiores de Monterrey (ITESM). (2016). Aprendizaje basado en investigación. Investigación e innovación educativa. Recuperado de http://sitios.itesm.mx/va/ dide2/tecnicas_didacticas/abi/copabi.htm

Kemmis, S. y Mctaggart, R. (1988). Cómo planificar la investigación-acción, Barcelona: Laertes.

Lapuebla, A. (1988). Una investigación colectiva. Cuadernos de Pedagogía, 157, 54 - 59. Recuperado de https://dialnet.unirioja.es/ servlet/articulo? codigo $=2770098$

Lewin, K. (1973). Action Research and Minority Problems. En R. Alexander \& J. Ellis (Eds.), Resolving Social Conflicts: Selected Papers on Group Dynamics (pp.201-210). London: Souvenir Press.

López-Górriz, I. (1993). La investigación-acción como metodología de teorización y formación del profesor desde su práctica.Revista de investigación educativa, RIE,10(20), 71-92. Recuperado de https://dialnet.unirioja.es/servlet/articulo?codigo $=764541$

Marcuzzo Do Canto O. (1996). El compromiso social de la educación superior. En J. Wainer (Ed.), La educación superior como responsabilidad de todos (pp. 33-35). Caracas: CRESALC/UNESCO.

Martínez, A. y Buendía, A. (2005). Aprendizaje basado en la investigación. Tecnológico de Monterrey. Recuperado de http://www.mty. itesm.mx/rectoria/dda/rieee/pdf-05/29(EGADE).A.BuendiaA. Mtz.pdf

Morales, O. Rincon, A. y Romero, J. (2004). Cómo enseñar a investigar en la universidad. Educere, 9(29), 217-224. Recuperado de www.saber.ula.ve/handle/123456789

Mosquera, J. C. yFurió-Más C. (2008). El cambio didáctico en profesores universitarios de química a través de un programa de actividades basado en la enseñanza por investigación orientada. 
Didáctica de las CienciasExperimentales y Sociales, 22, 115154. Recuperado de https://ojs.uv.es/index.php/dces/article/ view/2424

Peñaherrera, M., Chiluiza, K. y Ortiz, A. (2014). Inclusión del aprendizaje basado en investigación (ABI) como práctica pedagógica en el diseño de programas de postgrados en Ecuador. Elaboración de una propuesta. Journal for Educators, Teachers and Trainers, 5(2), 204-220. Recuperado de http://www.ugr.es/ jett/pdf/ Vol5(2)_015_jett_Penaherrera_Chiluiza_Ortiz.pdf

Restrepo-Gómez, B. (2005). Una variante pedagógica de la investigación-acción educativa. OEI-Revista Iberoamericana de Educación. Recuperado de http://www.rieoei.org/deloslectores/370Restrepo.PDF

Roach, M. Blackmore, P. y Dempster, J. (2001). Supporting High Level Learning Through Research-based Methods: A Framework for Course Development. Innovations in Education and Training International, 38(4) 369-382. Recuperado de http://www.tandfonline.com/doi/abs/10.1080/14703290110074957

Rodríguez-Paz, C. y Gijón, E. (2000). Investigación durante la licenciatura. Revista Facultad de Medicina de la UNAM, 45(5) 8789. Recuperado de http://www.medigraphic.com/pdfs/facmed/ un-2000/un003e.pdf

Rojas, G. (2009). La investigación como estrategia didáctica en la construcción del conocimiento escolar. Revista EDU-FÍSICA, 1-7. Recuperado de http://www.edu-fisica.com/Revista\%202/ INVESTIGACIONCOMO.pdf

Secretaría de Educación Pública (SEP). (2015). Sistema interactivo de consulta de estadística educativa. Recuperado de http://planeacion.sep. gob.mx/principalescifras/ consultado el 30 de julio de 2016.

Stenhouse, L. (1984). Investigación y desarrollo del currículo. Madrid: Morata.

Torres, A. (2012). Aprendizaje basado en la investigación. Técnicas didácticas. Tecnológico de Monterrey. Recuperado de www.itesca.edu.mx/ documentos/.../Metodo_Aprendizaje_Basado_en_Investigacion.p...

Universidad Nacional Autónoma de México (UNAM) (2012). Plan Educativo Nacional, Capitulo 8. Cobertura y estructura del sistema educativo mexicano: Problemática y propuestas. Recuperado de www.planeducativonacional.unam.mx/CAP_07/Text/07_02a.html 
Universidad de Quintana Roo (UQROO). (2013-2016). Plan Estratégico de Desarrollo Institucional 2013-2016. Recuperado de http://www.uqroo.mx/nuestra-universidad/documentos/ plan-estrategico-de-desarrollo-institucional-pedi-2013-16/

Universidad de Quintana Roo-Dirección General de Planeación. (UQROO-DGP). (s. f.). Sistema de Información de Gestión de la Calidad (SIGC). Recuperado de http://sigc.uqroo.mx/04_documentos_generales/modelo_educativo/modeloeducanew.pdf. 